\title{
"Ação a Distância versus Ação Intermediada. Contraste entre a Nova e as Velhas Visões sobre Eletricidade" - Tradução comentada de um texto de Oliver Heaviside sobre ação a distância
}

\author{
"Action at a Distance versus Intermediate Agency. Contrast of New with Old Views about Electricity" - A \\ commented Portuguese translation of a paper by Oliver Heaviside on action at a distance
Hugo Shigueo Tanaka dos Santos ${ }^{*}\left[\right.$, Daniel Gardelli ${ }^{2}$, Polônia Altoe Fusinato ${ }^{1}$, Marcos Cesar Danhoni Neves ${ }^{1,2}$
${ }^{1}$ Universidade Estadual de Maringá, Programa de Pós-Graduação em Educação para a Ciência e a Matemática, Maringá, PR, Brasil
${ }^{2}$ Universidade Estadual de Maringá, Departamento de Física, Maringá, PR, Brasil

\begin{abstract}
Recebido em 30 de setembro de 2019. Revisado em 01 de fevereiro de 2020. Aceito em 07 de fevereiro de 2020
Apresentamos uma tradução comentada de um texto de Oliver Heaviside publicado originalmente no livro Electromagnetic Theory, em 1893. Neste trabalho, Heaviside exibe seus argumentos a favor do éter luminífero. Palavras-chave: Oliver Heaviside, ação a distância, James Clerk Maxwell, ondas eletromagnéticas, Electromagnetic Theory.
\end{abstract}

A commented Portuguese translation of a text by Oliver Heaviside originally published in the book Electromagnetic Theory in 1893 is offered. In this work, Heaviside shows his arguments in favor of luminiferous ether.

Keywords: Oliver Heaviside, action at a distance, James Clerk Maxwell, electromagnetic waves, Electromagnetic Theory.

\section{Introdução}

Apresentamos a seguir um texto de Oliver Heaviside (1850-1925), intitulado Action at a Distance versus Intermediate Agency. Contrast of New with Old Views about Electricity [1] que foi publicado pela primeira vez em 1893, no primeiro volume do livro Electromagnetic Theory [1]. O texto é a introdução de um capítulo no qual Heaviside discute a teoria das ondas eletromagnéticas planas. Aqui apresentamos a versão do texto que está disponível na edição de 1971 da obra citada.

Na obra Electromagnetic Theory, Oliver Heaviside "removeu os obstáculos para a criação de um 'Novo eletromagnetismo', em que os princípios fundamentais da teoria de Maxwell seriam expostos com clareza e percepção sem precedentes" [2, p. 250].

Heaviside se propõe a mostrar o contraste entre dois mecanismos propostos sobre a interação entre corpos: a ação a distância e a ação intermediada. Assim como James Clerk Maxwell (1831-1879), ele também leva em consideração as teorias de ação a distância 1

\footnotetext{
*Endereço de correspondência: hshigueo@gmail.com

1 O texto de Maxwell sobre as teorias de ação a distância já se encontra traduzido para o português por [3].
}

No texto de Heaviside traduzido neste artigo, o cientista inglês discute conceitos e contrasta as duas teorias de interação entre corpos, sem entrar em conceitos matemáticos. Porém, o autor não expõe os argumentos favoráveis à ação a distância, trazendo argumentos favoráveis apenas à sua visão: a da ação mediada. Para tanto, o autor faz analogia com a propagação de ondas sonoras. As analogias físicas eram muito utilizadas nos modelos científicos do século XIX a fim de tornar as teorias mais compreensíveis, justificáveis e utilizáveis na prática [4-7]. Nessa corrente de pensamento "[...] o propósito de se elaborar modelos analógicos era o de se familiarizar com determinado campo de conhecimento, a ponto de ser possível descrevê-lo por meio de sua própria linguagem [...]" [8, p. 268].

O presente trabalho é parte de uma pesquisa de mestrado em Educação para a Ciência e a Matemática. Acreditamos que o uso da História da Ciência no Ensino, principalmente em uma abordagem que explicite as controvérsias que ocorrem durante a construção das Ciências, seja capaz de formar cidadãos críticos, que os levem a conhecer a riqueza da construção das Ciências e a serem 
capazes de questionar de maneira sensata as afirmações feitas por especialistas $2^{2}$

\section{Oliver Heaviside, uma breve biografia}

Oliver Heaviside foi um engenheiro elétrico inglês, nascido em 18 de maio de 1850, durante o período vitoriano, em uma família de baixo poder aquisitivo. Por conta disso, não recebeu educação formal após os dezesseis anos. Heaviside " [...] era um homem muito complexo, difícil de lidar. Ele era um indivíduo inseguro, insensível e grosseiro. Mas, também era muito mais. Era um físicomatemático de primeira, um engenheiro elétrico com incríveis habilidades analíticas e inspiração física" [15, p. 1-2]. Nunca ocupou um cargo acadêmico e, na maior parte da vida, não teve emprego. Por trabalhar de forma independente e, na maior parte do tempo, sem salário, Heaviside é considerado o último cientista amador [2].

A tia por parte de mãe de Heaviside casou-se com Charles Wheatstone (1802-1875), que já era um cientista conhecido àquela época. A influência e a proximidade com Wheatstone teve grande influência sobre os integrantes da família Heaviside, principalmente em Oliver. Por influência do tio, Oliver acabou trabalhando como operador de telégrafo na Dinamarca, influenciando-o, desta forma, em suas pesquisas [15].

O primeiro artigo de Heaviside data de julho de 1872, intitulado Comparing electromotive forces (Comparando forças eletromotrizes), no qual ele descrevia um método simples de comparar forças eletromotrizes [16], que fora concebido por ele em 1870. Em fevereiro de 1873, Heaviside [16] publicou seu segundo artigo, intitulado $O n$ the Best Arrangement of Wheatstone's Bridge for Measuring a Given Resistance with a Given Galvanometer and Battery ( $O$ melhor arranjo da ponte de Wheatstone para a medição de uma dada resistência com um dado galvanômetro e uma bateria), no qual usa cálculo diferencial para discutir uma nova configuração da ponte de Wheatstone. Devido a um conselho de William Thomson (1824-1907) - que mais tarde receberia o título de Lord Kelvin - Heaviside enviou uma cópia de seu artigo para Maxwell, que o citou a partir da segunda edição de sua obra A Treatise on Electricity and Magnetism (Um Tratado sobre Eletricidade e Magnetismo) [17-18].

Não se sabe ao certo, qual era o nível de conhecimento físico e matemático de Heaviside ao ler pela primeira vez a obra de Maxwell, em 1873, logo após sua primeira publicação, mas é certo que esta obra o influenciou. Com o tempo, Heaviside se tornou o mais influente estudante da teoria de Maxwell e foi ele quem mais contribuiu para que as 20 equações propostas por Maxwell no Treatise

\footnotetext{
2 A importância da História da Ciência para o Ensino de Ciências já foi apontada por vários pesquisadores. Para uma leitura inicial sobre o assunto, pode-se consultar [9-14]
}

fossem adaptadas para o que é conhecido atualmente como "as quatro equações de Maxwell' [3]2].

A partir de 1885, Heaviside passou a publicar trabalhos tentando reformular a teoria de Maxwell. Neste período, alguns conceitos novos surgiam, depois eram esquecidos e reapareciam anos depois [19]. Após 1900, a produção científica de Heaviside teve um forte declínio devido, principalmente, a problemas de saúde. Lentamente, até sua morte, em 1925, ele se tornou mais recluso e excêntrico $[2]$.

As contribuições de Oliver Heaviside para a Física e a Engenharia são diversas, porém, pouco discutidas atualmente. Algumas destas contribuições são mostradas por Tonidandel e Araújo [20] e Menon [21].

\section{Contexto histórico}

No que diz respeito à interação entre eletricidade e magnetismo, consideramos como marco inicial a observação do fenômeno da deflexão de uma agulha magnetizada ao ser aproximada de um fio condutor com corrente, previsto por Hans Christian Ørsted (1777-1851), no ano de 1812 e observado pelo mesmo cientista em 1820 [22]. O experimento fez com que diversos estudiosos da época tentassem explicar a interação. Dentre eles, destacamos: AndréMarie Ampère (1775-1836), Michael Faraday (1791-1867), Jean-Baptiste Biot (1774-1862), Félix Savart (1791-1841) e o próprio Ørsted. A descrição mais detalhada de cada uma dessas interpretações podem ser encontradas em [23] e [24]. Pode-se, brevemente, sintetizar as quatro das principais interpretações dadas ao experimento de Ørsted da seguinte maneira, conforme apontam Santos e Fusinato [25]:

1. Biot e Savart concluíram que o torque magnético gerado pela interação entre o fio e a agulha, deveria variar com o inverso da distância. Além disso, para a dupla, a interação entre a bússola e o fio com corrente, deveria ser puramente magnética. Isto é, não admitiam a existência de campos magnéticos gerados por correntes elétricas e sim que o próprio fio, ao ser percorrido por corrente elétrica, magnetizava-se de uma maneira especial, e passava a atuar como um ímã.

2. Para Faraday, a natureza da força eletromagnética deveria ser circular. Caso um dos polos ímã pudesse ser isolado, ele giraria em torno do fio. Para Faraday, a interação entre os corpos deveria acontecer por meio de linhas de força, as quais podem ser visualizadas ao se espalhar limalhas de ferro próximas a um fio com corrente.

3. Ørsted interpretou seu experimento como sendo devido ao surgimento de duas correntes elétricas que tentavam se movimentar em sentidos opostos no interior do fio e por isso, gerariam uma espécie de conflito elétrico, que por sua vez, daria origem a turbilhões de matéria elétrica, os quais faziam espirais em sentidos opostos em

\footnotetext{
${ }^{3}$ Recomendamos a leitura do apêndice intitulado Das equações de Maxwell às "equações de Maxwell", disponível em [2, p. 383-385].
} 
torno do fio e que interagiam com a agulha da bússola próxima a ele. Tal interpretação se assemelhava à maneira como Descartes interpretava o movimento planetário.

4. Para interpretar o fenômeno chamado por Ørsted de conflito elétrico, e que supostamente estaria ocorrendo no interior do fio, Ampère elaborou um programa de pesquisa completamente novo e que se mostrou extremamente frutífero [24], porque o físico francês foi capaz de prever e observar vários fenômenos inéditos até aquela época.

Além disso, Ampère chegou a uma equação para a interação entre elementos de corrente que atualmente é conhecida como Força entre elementos de corrente de Ampère, que, em notação matemática contemporânea, como mostrado por Assis [26] é da forma:

$d^{2} \vec{F}_{12}^{A}=\frac{\mu_{0}}{2 \pi} \frac{i_{1} i_{2}}{r_{12}^{2}}\left[\left(d \vec{l}_{1} \cdot d \vec{l}_{2}\right)-\frac{3}{2}\left(d \vec{l}_{1} \cdot \widehat{r}_{12}\right)\left(d \vec{l}_{2} \cdot \widehat{r}_{12}\right)\right] \widehat{r}_{12}$ (1)

Essa equação fornece um resultado igual ao da Lei de Biot-Savart e da Força de Grassmann para a interação entre dois fios com corrente. A equivalência é ilustrada no trabalho escrito por Santos e Gardelli [27].

A força entre elementos de corrente de Ampère foi chamada por Maxwell de equação cardeal da eletrodinâmica [18, artigo 528, p. 175], o que explicita o fato de que esta equação deveria ser considerada como a equação fundamental de toda a eletrodinâmica. Porém, nos dois volumes de sua obra $A$ Treatise on Electricity and Magnetism [17-18], o autor não utiliza um tratamento eletrodinâmico, ou seja, de interação entre elementos de corrente elétrica para analisar os fenômenos que estavam sendo estudados. Ele apresenta uma nova teoria que, por sua vez, foi influenciada pela escola inglesa e pela busca de análogos mecânicos para as interações físicas [28-29].

Ainda assim, Maxwell presta um grande tributo às teorias de ação a distância, conforme o prefácio à primeira edição do seu Treatise:

Grande progresso tem sido feito um na ciência elétrica, principalmente na Alemanha, pelos cultivadores da teoria de ação a distância. As valiosas medições elétricas de $W$. Weber são interpretadas por ele, de acordo com sua teoria, e a especulação eletromagnética que foi originada por Gauss, e continuada por Weber, Riemann, J. e C. Neumann, Lorenz etc., está baseada na teoria de ação a distância, mas dependendo ou diretamente da velocidade relativa das partículas, ou da propagação gradual de alguma coisa, seja potencial ou força, de uma partícula à outra. O grande sucesso que esses célebres homens alcançaram na aplicação da matemática aos fenômenos elétricos fornece, como é natural, peso adicional às suas especulações teóricas, de tal forma que aqueles que, como estudantes da eletricidade, se voltam em direção a eles como as maio- res autoridades em eletricidade matemática, provavelmente assimilariam, junto com seus métodos matemáticos, suas hipóteses físicas 4 [17, p. x-xi, tradução nossa].

Além disso, a segunda edição do Treatise de Maxwell tem o último capítulo inteiramente dedicado às teorias de ação a distância. Este capítulo discorre sobre argumentos a favor e contra teorias de ação a distância e teorias de ação por campos. A tradução comentada desse trabalho pode ser encontrada em [3].

Apesar disso, Oliver Heaviside foi um dos seguidores de Maxwell responsáveis por consolidar o seu legado e, também, por resumir a apenas quatro as vinte equações originais propostas no Treatise.

Em um artigo de 1888, que foi reproduzido no Electrical Papers, sobre a equação de Ampère, Heaviside disse:

Foi afirmado, em não menor autoridade do que o grande Maxwell, que a lei de força entre um par de elementos de corrente de Ampère é a fórmula cardeal da eletrodinâmica. Se é assim, não deveríamos usá-la sempre? Nós alguma vez a usamos? Maxwell a utilizou no seu Tratado [A Treatise on Electricity \& Magnetism]? Certamente há algum erro. Isto não significa que eu queira roubar de Ampère o crédito por ser o pai da eletrodinâmica. Eu só transferiria o nome de fórmula cardeal para outra [fórmula], devido a ele [Ampère], expressando a força mecânica sobre um elemento [de corrente] de um condutor de eletricidade [que se encontra] em [uma região do espaço onde exista] um campo magnético qualquer; [ou seja, expressando] o produto vetorial entre a corrente e a indução [magnética]. Há algo real sobre isso. Não é como sua força [força de Ampère] entre um par de elementos abertos; isso é fundamental; e, como todos sabem, ainda está em uso, efetivamente ou virtualmente (por meio da força eletromotriz) por [físicos] teóricos ou experimentais 5 [30, p. 501-502, tradução nossa].

\footnotetext{
4 No original, "Great progress has been made in electrical science, chiefly in Germany, by cultivators of the theory of action at a distance. The valuable electrical measurements of W. Weber are interpreted by him according to this theory, and the electromagnetic speculation -which was originated by Gauss, and carried on by Weber, Riemann, J. and C. Neumann, Lorenz, \&c., is founded on the theory of action at a distance, but depending either directly on the relative velocity of the particles, or on the gradual propagation of something, whether potential or force, from the one particle to the other. The great success which these eminent men have attained in the application of mathematics to electrical phenomena, gives, as is natural, additional weight to their theoretical speculations, so that those who, as students of electricity, turn to them as the greatest authorities in mathematical electricity, would probably imbibe, along with their mathematical methods, their physical hypotheses." [17, p. x-xi].

5 No original, "It has been stated, on no less authority than that of the great Maxwell, that Ampère's law of force between a pair of current-elements is the cardinal formula of electrodynamics. If
} 
Ou seja, Heaviside deseja transferir o título de equação cardeal da eletrodinâmica para outra equação de Ampère. Porém, Heaviside dá uma interpretação completamente distinta de Ampère, visto que o cientista francês nunca admitiu a existência de campos magnéticos. Como apontado por [31], a força devido a um circuito fechado em um elemento de corrente, foi elaborada pela primeira vez por Ampère, na qual é admitida a existência de uma diretriz. Tal diretriz é um fator puramente geométrico e depende apenas da forma do circuito. Apenas ao multiplicar essa diretriz pela corrente elétrica, é que, posteriormente, se atribuiu a ela um significado físico.

Outro ponto que vale a pena mencionar, é o fato de se atribuir a Oliver Heaviside a obtenção da equação do telégrafo, como apontado por Whittaker [32, p. 228-229]. Porém, a solução da mesma equação já havia sido encontrada de maneira independente por Gustav Kirchhoff (1824-1887) e Wilhelm Eduard Weber (1804-1891) quase vinte anos antes de Heaviside. Ambos os cientistas chegaram ao mesmo resultado utilizando os conceitos da eletrodinâmica de Weber [33-34] a qual admite apenas a ações a distância.

\section{Tradução}

Após esta breve introdução, apresentaremos a seguir a tradução comentada deste texto importante para a História da Física. $\{306\}\}^{6}$

\section{CAPÍTULO IV}

\section{TEORIA DAS ONDAS ELETROMAGNÉTICAS PLANAS}

Ação a Distância versus Ação Intermediada. Contraste entre a Nova e as Velhas Visões sobre Eletricidade

\$176 Muitas vezes tem sido observado que o universo está em uma condição instável. Nada está imóvel. Nem podemos manter o movimento, uma vez produzido, para uma determinada quantidade de matéria. Ele é difuso ou de outra forma é transferido para outra matéria, seja imediatamente ou eventualmente. O mesmo fato é observado nos mundos moral e intelectual assim como no material, mas isso só nos preocupa a ponto de dizer que

so, should we not be always using it? Do we ever use it? Did Maxwell, in his treatise? Surely there is some mistake. I do not in the least mean to rob Ampère of the credit of being the father of electrodynamics; I would only transfer the name of cardinal formula to another due to him, expressing the mechanical force on an element of a conductor supporting current in any magnetic field; the vector product of current and induction. There is something real about it; it is not like his force between a pair of unclosed elements; it is fundamental; and, as everybody knows, it is in continual use, either actually or virtually (through electromotive force) both by theorists and practicians[30, p. 501-502].

6 Os números entre chaves $(\{\})$ representam a paginação original do primeiro capítulo do Electromagnetic Theory, assim como mostrado em [1] ele subjaz à comunicação do conhecimento aos outros quando o espírito se move, embora a tarefa seja de uma natureza ingrata 7

As leis pelas quais os movimentos, ou fenômenos que dependem em última instância do movimento, são transferidos naturalmente formam um importante objeto de estudo dos físicos. Existem duas visões principais extremas sobre o processo. Há a teoria da ação a distância instantânea entre corpos diferentes sem um agente intermediáric 8 e, por outro lado, há a teoria da propagação no tempo através e por meio de um agente intermediário. No último caso, os corpos distantes não atuam realmente um sobre o outro, mas apenas parecem fazê-lo. Na verdade, eles agem diretamente sobre um meio, e entre os dois há a ação entre partes contíguas do próprio meio. Mas, no primeiro caso, a ideia de um meio não é levada em consideração. Nós podemos, entretanto, de alguma forma, modificar a visão para que a ação, embora aparentemente instantânea e direta, ocorra através de um agente intermediário, sendo a velocidade de transmissão tão grande que está além da compreensã $q^{9}$ Assim, as duas

$\{307\}$

ideias de ação direta, e por um agente intermediário, podem ser de alguma forma conciliadas, sendo feitos casos extremos de uma teoria. Por exemplo, se nós sabemos que há um agente intermediário e uma velocidade finita, mas em um certo caso em análise a influência desse agente não é sentida, então nós praticamente podemos assumir que a velocidade é infinita. Mas, embora isso seja a mesma coisa que a ação a distância, nós não precisamos ir além e acabar com a ideia de um agente intermediário ${ }^{10}$

Há outra maneira de considerar o assunto. Nós podemos explicar a propagação no tempo através de um meio por ações a distância. Mas isso é inútil como explicação, sendo, na melhor das hipóteses, meramente a expressão de uma equivalência matemática ${ }^{11}$

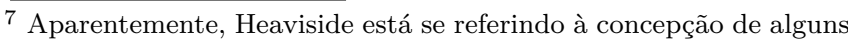
filósofos de que o conhecimento transmitido causa o movimento do espírito universal. Tal transmissão de conhecimentos (ou de informação) é a ação mediada no mundo das ideias.

8 A fim de facilitar a compreensão do texto, optamos por traduzir "intervening medium" como agente intermediário.

9 Aqui Heaviside, aparentemente, propõe uma adaptação dos conceitos da ação a distância para uma ação por campos com velocidade próxima do infinito.

10 Aqui há um erro de lógica de Heaviside. Ação a distância não é a mesma coisa que uma ação mediada com velocidade infinita. A ação a distância, para seus defensores, é a interação simultânea entre corpos. Além disso, se não há um meio intermediário, não faz sentido falar em velocidade de perturbação nele, seja essa velocidade infinita ou não.

11 As bases conceituais da ação a distância e da ação por campos são distintas. Apesar de o resultado final em ambos os tratamentos ser equivalente, existem grandes diferenças. Por exemplo, para a interação entre fios condutores, ao utilizar o eletromagnetismo clássico (ação por campos), a força entre os elementos de corrente não obedece à terceira lei de Newton e só o fazem quando se considera todo o circuito. Já em um tratamento weber-amperiano (ação a distância), o princípio da ação e reação sempre é satisfeito. Para uma melhor compreensão, sugerimos a leitura de [27].
} 
Agora considere a transmissão do som. Isso consiste, fisicamente, de movimentos vibratórios de matéria, e é transmitido por ondas no ar e nos corpos imersos nele. A velocidade [do som] no ar é bem pequena, tão pequena que nem sequer pode deixar de ser notada pelos antigos, que eram, em geral, decididamente não-científicos nas suas atitudes mentais em relação aos fenômenos naturai ${ }^{12}$. Suponha, no entanto, que a velocidade de transmissão do som no ar seja muitas vezes maior do que realmente é, de modo que uma ideia de velocidade infinita, ou de uma velocidade por si só, não esteja presente. Devemos então ter os fatos principais diante de nós, que os corpos materiais podem vibrar de acordo com certas leis, e que eles podem, além disso, colocar corpos distantes em vibração. Isso poderia ser a introdução de vibrações, e poderia ser explicado, na ausência de um conhecimento melhor, por meio da ação a distância de matéria agindo sobre matéria, em última análise solucionável em alguma forma da lei do inverso do quadradq 13 . não porque há alguma coisa essencialmente acústica sobre isso, mas por causa das propriedades do espaç 14 Além disso, nós sempre deveríamos, naturalmente, associar som com os corpos limitados pelo ar, mas não com o próprio ar, que não entraria na teoria. Um filósofo eruditc 15 que devesse explicar as questões em termos de um meio intermediário, poderia não ver suas ideias serem rapidamente aceitas, embora ele apontasse evidência independente para a existência do meio - fisiológica e mecânica - e houvesse uma harmonia de propriedades essenciais envolvidas ${ }^{16}$ Nada menos que a prova real da velocidade finita do som convenceria os preconceituosos.

$\{308\}$

Agora, embora exista, indubitavelmente, uma grande diferença nos detalhes entre a transmissão do som e [a transmissão] de distúrbios elétricos, mesmo assim há

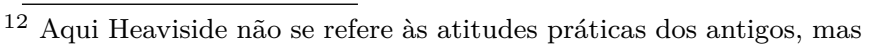
de atitudes mentais, o que aparenta se referir à matemática. Porém, diversos filósofos naturais da Antiguidade tiveram muito êxito sem se preocuparem necessariamente com uma descrição quantitativa dos fenômenos, como Arquimedes, João Filopono, entre outros.

13 A lei do inverso do quadrado, a saber, é uma relação de proporcionalidade que é da forma: $\mathrm{F} \propto 1 / \mathrm{r}^{2}$, isto é, a "força" irá diminuir com o quadrado da distância entre os dois corpos interagentes. Um exemplo conhecido desta relação de proporcionalidade é a lei da gravitação universal de Newton.

14 Novamente há um equívoco de Heaviside ao dizer que os defensores da ação a distância atribuem a lei do inverso do quadrado às propriedades do espaço. Ora, se a ação ocorre a distância, ela não precisa do "espaço" com propriedades. Para os defensores da ação a distância, a física vem antes da matemática, dessa forma, a lei do inverso do quadrado existe porque a física das interações é assim. $\mathrm{E}$, se por um lado, os defensores do éter usam o meio como um argumento de a queda da intensidade da força ser análoga à queda de intensidade de uma onda esférica ao se propagar $\left(1 / \mathrm{r}^{2}\right)$, o mesmo não se sustenta para algumas forças de ação a distância, cuja força depende de posições, velocidades e acelerações relativas entre os corpos interagentes, como na força de Weber, por exemplo.

15 No original: "deepminded philosopher".

16 Provavelmente, Heaviside está fazendo uma analogia com o fato de que a teoria de Maxwell demorou a ser aceita. Na época, os conceitos maxwellianos eram considerados desajeitados e confusos. $[2]$. uma semelhança suficientemente ampla para se fazer com que o caso suposto acima seja análogo ao que realmente acontece na ciência do eletromagnetismo. Há condutores e não-condutores, ou isolantes, e como a velocidade finita de propagação no espaço não-condutor fora dos condutores era desconhecida, a atenção era quase toda concentrada nos condutores e num suposto fluido que deveria residir sobre eles ou neles, e se mover sobre eles ou através deles. E a influência sobre condutores distantes era atribuída à ação a distância instantânea, ignorando um agente intermediário. Novamente, um filósofo erudito elaborou uma teoria para explicar estas interações por ação intermediada de um meio transmitindo a uma velocidade finita ${ }^{17}$ Mas, ao fazê-lo, ele utilizou o mesmo meio cuja existência já havia evidência independente, isto é, o éter luminífer ${ }^{18}$ e apontou a consistência das propriedades essenciais requeridas nos dois casos, e que sua teoria eletromagnética fez até uma teoria da luz muito melhor do que a antiga ${ }^{19}$ ainda que suas visões não tenham se espalhado rapidamente. As antigas visões persistiram, apesar da probabilidade intrínseca da nova, e apesar da grande quantidade de evidências em apoio à visão de algum meio fora dos condutores, e que podem estar dentro também, mas não particularmente conduzindo matéria propriamente dita, estava essencialmente interessado nos fenômenos elétrico: 20 valor e a validade das evidências variam de acordo com o estado da mente do julgador. Para aqueles que tinham estudado seriamente a teoria de Maxwell, a evidência a seu favor era irresistível e esmagadora; outros não acreditavam nela nem um pouco. Estou, no entanto, inclinado a pensar que teria prevalecido antes, por muito tempo, mesmo que não houvesse evidência direta da velocidade finita. Era simplesmente uma questão de tempo. Mas a prova experimental da velocidade finita de transmissão estava por vir, e a influência muito lenta do pensamento teórico dos conservadores foi reforçada pelo apelo do senso comum dos fato\$21. Agora é um

$17 \overline{\text { Aqui Heaviside se refere, }}$ de fato, a Maxwell. Além do que foi dito na nota anterior, vale destacar também, que em 1879, quando Maxwell morreu, apenas poucos de seus contemporâneos haviam sido convencidos de sua teoria [2].

18 Maxwell desenvolveu sua teoria levando-se em consideração o éter luminífero como agente intermediário da interação, porém, hoje em dia, as interações são estudadas como se o vácuo fosse o meio de interação física, conforme apontado por[23].

19 Novamente, Heaviside se refere a Maxwell. Porém, é necessário relembrar que a velocidade da luz já havia sido calculada e medida experimentalmente por Weber e Kohlrausch entre os anos de 1855 e 1856 [35].

20 O princípio que norteou a revisão da teoria maxwelliana foi o conceito do fluxo de energia. A ideia de energia se movendo através do campo eletromagnético foi formulada primeiramente por John Henry Poynting (1852-1914), no fim de 1883. Heaviside, de maneira independente, também postulou este princípio meses mais tarde $[2]$.

21 Aqui, por "conservadores", Heaviside se refere àqueles cientistas que custavam a aceitar a ideia de que as interações físicas entre eletricidade e magnetismo eram o resultado da interação por campos. Isto é, para Heaviside, acreditar em uma ação a distância entre eletricidade e magnetismo seria uma ideia conservadora. Apesar disso, a partir das concepções de Ampère para a interação entre 
fato que as ondas eletromagnéticas se propagam fora dos condutores assim como as ondas sonoras se propagam fora de corpos vibrantes ${ }^{22}$ É uma inferência científica legítima que há um meio

\section{$\{309\}$}

para fazê-lo tanto em um caso como no outro, e há evidência independente a favor de ambos os meios, ar para o som e éter para as perturbações elétricas. Também é muito provável agora que as vibrações da luz nada mais são que vibrações eletromagnéticas muito rápidas, que eu penso que essa visão prevalecerá inteiramente, mesmo que a lacuna que existe entre as vibrações hertzianas e as vibrações da luz não seja preenchida experimentalmente pela falta de aparelhos adequados - desde que alguma descoberta completamente nova de caráter imprevisto, não é loucura pensar que isso irá refutar a possibilidade da identidade assumida.

Agora, a questão imediata aqui é como propagar um conhecimento sobre a teoria das ondas eletromagnéticas 23 Se pudéssemos assumir que o leitor tivesse tido um bom treinamento matemático, isso facilitaria muito o assunto. Por outro lado, dificilmente será útil tentar fazê-lo para aqueles que não tenham conhecimento matemático menos ainda para aqueles agressores anti-matemático ${ }^{24}$ da teoria da propagação de ondas de perturbações elétricas que mostram plenamente que eles sequer sabem de forma inteligível o que uma onda significa, e o que sua existência implica. Mas entre os dois, eu acho que é possível fazer um bom acordo no modo de propagação por meio de uma exposição detalhada da teoria das ondas planas, especialmente nos dielétricos. Com o uso de ondas planas, a complexidade matemática das ondas em geral desaparece em grande medida, e a álgebra comum pode ser substituída largamente pela análise que ocorre em casos mais avançados. A maioria das propriedades essenciais e ideias podem ser assimiladas por um leitor pensante que não é avançado em sua matemática, e o que está além dele, ele pode pular. Além disso, o próprio

elementos de corrente, Weber e Kirchhoff calcularam, de maneira independente, pela primeira vez a velocidade da luz e resolveram uma equação de onda [36; 32-33].

22 Aqui Heaviside argumenta que as vibrações eletromagnéticas dentro de um fio seriam da mesma natureza que as ondas descobertas por Hertz as quais, por sua vez, seriam da mesma natureza que a luz. Vale destacar que à época, não estava posta a identidade entre esses fenômenos. Ironicamente, parece que foram os experimentos de Hertz que evidenciaram o efeito fotoelétrico pela primeira vez. 23 O objetivo de Heaviside era, declaradamente, ensinar os princípios do eletromagnetismo de Maxwell da maneira mais clara e simples que fosse capaz. Hoje em dia é usado o tratamento vetorial quando se estuda eletromagnetismo. Isso acontece, principalmente, devido aos esforços de Heaviside e outros [2]. Esta preocupação em facilitar a compreensão da teoria eletromagnética pode ser notada em seus artigos, compilados no Electrical Papers [16] e no terceiro capítulo do Electromagnetic Theory [1].

24 Novamente Heaviside se refere aos filósofos naturais que não se preocupavam com uma descrição quantitativa dos fenômenos como "anti-matemáticos" e não-científicos. Porém, é importante notar que o termo "cientista" só se popularizou depois da virada do século XIX. tratamento das ondas planas é a melhor preliminar para casos mais gerais.

Os problemas que se apresentam por limitação artificial às ondas planas são muitas vezes de natureza abstrata. Há, entretanto, algumas exceções importantes; sendo a mais notável aquela da propagação ao longo de fios retos, cuja teoria é essencialmente a das ondas planas, modificadas pela resistência dos fios. Antes, entretanto, procedendo para os detalhes das ondas planas, que constituirão o tema deste capítulo (embora seja desnecessário excluir completamente os assuntos conexos), será desejável preparar a mente para

$\{310\}$

um esboço de algumas noções gerais sobre ondas eletromagnéticas, independentemente do tipo preciso dela.

\section{Material Suplementar}

O seguinte material suplementar está disponível online:

ANEXO - Action at a Distance versus Intermediate Agency. Contrast of New with Old Views about Electricity.

\section{Referências}

[1] O. Heaviside, Electromagnetic Theory (Chelsea Publishing, New York, 1971).

[2] B.J. Hunt, Os seguidores de Maxwell (Editora UFMG, Belo Horizonte, 2015).

[3] A.C. Tort, A.M. Cunha e A.K.T. Assis, Revista Brasileira de Ensino de Física 26, 3 (2004)

[4] K.F. Schaffner, Nineteenth-Century Aether Theories (Pergamon Press, New York, 1972).

[5] G.N. Cantor e M.J.S. Hodge (eds) Conceptions of ether: Studies in the history of ether theories - 1740-1900 (Cambridge University Press, Cambridge, 1981).

[6] P.M. Harman, Energy, Force, and Matter - The Conceptual Development of Nineteenth-Century Physics (Cambridge University Press, Cambridge, 1982).

[7] P. Duhem, A Teoria Física: Seu Objeto e Sua Estrutura (EdUERJ, Rio de Janeiro, 2014).

[8] D.A.V. Tonidandel, W.C. Boaventura e A.E.A. Araújo, Revista Brasileira de História da Ciência 11, 2 (2018).

[9] S.G. Brush, Science 183, 4130 (1974).

[10] R.A. Martins, Boletim da Sociedade Brasileira de História da Ciência 9, 3 (1990).

[11] R.A. Martins, em: Estudos de história e filosofia das ciências: subsídios para aplicação no ensino, editado por C.C. Silva (Livraria da Física, São Paulo, 2006).

[12] M.R. Matthews, Science Teaching - The Role of History and Philosophy of Science (Routledge, New York, 1994).

[13] M.R. Matthews, Caderno Catarinense de Ensino de Física 12, 3 (1995).

[14] M.C.D. Neves, Revista Ciência \& Educação 5, 1 (1998).

[15] P.J. Nahin, Oliver Heaviside: Sage in Solitude: The Life, Work, and Times of an Electrical Genius of the Victorian Age (IEEE Press, London, 1987).

[16] O. Heaviside, Electrical Papers (Macmillan and Co., London, 1892), v. 1. 
[17] J.C. Maxwell, A Treatise on Electricity \& Magnetism (1873) (Dover, New York, 1954a), v. 1.

[18] J.C. Maxwell, A Treatise on Electricity \& Magnetism (1873) (Dover, New York, 1954b), v. 2.

[19] J. Z. Buchwald, Centaurus 28, 288 (1985).

[20] D.A.V. Tonidandel e A.E.A. Araújo, Revista Brasileira de Ensino de Física 37, 3 (2015).

[21] M.J. Menon, Revista Brasileira de Ensino de Física 31, 2 (2009).

[22] R.A. Martins, Cadernos de História e Filosofia da Ciência 10, 89 (1986).

[23] D. Gardelli, Concepções de Interação Física: Subsídios para uma Abordagem Histórica do Assunto no Ensino Médio. Dissertação de Mestrado, Universidade de São Paulo, São Paulo (2004).

[24] A.K.T. Assis e J.P.M. Chaib, Eletrodinâmica de Ampère - Análise do significado e da evolução da força de Ampère, juntamente com a tradução comentada de sua principal obra sobre eletrodinâmica (Editora Unicamp, Campinas, 2011).

[25] H.S.T. Santos e P.A. Fusinato, em Anais do V Simpósio Nacional De Ensino De Ciência e Tecnologia, Ponta Grossa, 2016 (UTFPR, Ponta Grossa, 2016).

[26] A.K.T. Assis, Eletrodinâmica de Weber - Teoria Aplicações e Exercícios (Editora Unicamp, Campinas, 1995).

[27] H.S.T. Santos e D. Gardelli, Caderno Brasileiro de Ensino de Física 34, 3 (2017).

[28] P. Duhem, Ciência e Filosofia 4, 63 (1989).

[29] P.C.C. Abrantes, Imagens de Natureza, Imagens de Ciência (Papirus, Campinas, 1996).

[30] O. Heaviside, Electrical Papers (Macmillan and Co., London, 1894), v. 2.

[31] E.T. Whittaker, A History of the Theories of Aether and Electricity (Tomash Publishers, New York, 1951), v. 1.

[32] J.C. Poggendorff, arXiv:1912.05930.

[33] J.A. Hernandes e A.K.T. Assis, em Anais do XXI Encontro Nacional de Física de Partículas e Campos, São Lourenço, 2000 (SBF, São Lourenço, 2000), p. 1-6.

[34] A.K.T. Assis, em Anais do VII Seminário Nacional de História da Ciência e da Tecnologia, São Paulo, 1999, editado por J.L. Goldfarb e M.H.M Ferras (Edusp, São Paulo, 2000), p. 65-71.

[35] A.K.T. Assis, em: Instantaneous Action at a Distance: "Pro" and "Contra", editado por A.E. Chubykalo, V. Pope e R. Smirnov-Rueda (Nova Science Publishers, New York, 1999). 